\title{
A COMPARISON OF THE EFFECTS OF LNOR-EPINEPHRINE, SYNTHETIC $L$-EPINEPHRINE, AND U.S.P. EPINEPHRINE UPON CEREBRAL BLOOD FLOW AND METABOLISM IN MAN ${ }^{1}$
}

\author{
BY WILLIS SENSENBACH, LEONARD MADISON, AND LAMAR OCHS
}

(From the Department of Medicine, Southwestern Medical School of the University of Texas, and the Department of Medicine, Veterans Administration Hospital, Dallas, Texas)

(Submitted for publication November 17, 1952; accepted December 10, 1952)

Nor-epinephrine, a pressor amine known to exist normally in the mammalian body, is identical with epinephrine except for the absence of a methyl group in its chemical structure. Since methylation occurs readily in the body, it has been suggested that nor-epinephrine functions chiefly as a precursor to epinephrine (1-5). Nevertheless, it possesses potent pharmacologic properties which differ in many respects from those of epinephrine (6-9). Epinephrine raises systolic blood pressure, increases cardiac output and accelerates the heart rate; it constricts blood vessels in certain areas of the circulatory bed, dilates them in others, with an over-all effect of dilatation and a decrease in total peripheral vascular resistance. Nor-epinephrine increases systolic and diastolic blood pressure, has little or no effect on cardiac output and decreases cardiac rate. It constricts blood vessels in all areas of the circulation in which its effect has been studied.

Little is known, however, of the effects of norepinephrine and epinephrine upon the cerebral circulation in man. A clear understanding of their effects on this important segment of the circulation should provide information bearing on a number of physiologic, pathologic, and therapeutic questions. Because of the similarity of the hemodynamic changes in essential hypertension to those induced experimentally by the administration of $l$-nor-epinephrine, it has been suggested that $l$-norepinephrine may play an important role in the pathogenesis of essential hypertension (6). A measure of support for this hypothesis would be gained if nor-epinephrine-induced hypertension

1 Reviewed in the Veterans Administration and published with the approval of the Chief Medical Director. The statements and conclusions published by the authors are the result of their own study and do not necessarily reflect the opinion or policy of the Veterans Administration. could be shown to be associated with changes in the cerebral circulation similar to those accompanying essential hypertension. If, on the other hand, $l$-nor-epinephrine affects the cerebral circulation in some unique or distinctive way, this information might be of some value in the clinical recognition and detection of certain hypertensive states associated with abnormally high concentrations of $l$-norepinephrine in the adrenal gland, i.e., pheochromocytoma $(10,11)$. Furthermore, nor-epinephrine has been employed therapeutically as a pressor agent in a variety of conditions characterized by acute hypotension including operative, hemorrhagic, and traumatic shock; to maintain blood pressure after thoracolumbar sympathectomy; and in the circulatory collapse that may accompany acute myocardial infarction $(12,13)$. A clear understanding of its effect on the cerebral circulation would be essential to a proper assessment of its over-all therapeutic value in these conditions. Obviously, if the increase in systemic arterial blood pressure is accompanied by a disproportionate increase in cerebral vascular resistance, a further reduction in blood flow to the brain would ensue. Such an effect would, no doubt, detract from its usefulness in the treatment of these hypotensive states.

This report deals with studies made to determine the effect of $l$-nor-epinephrine, and $l$-epinephrine on the cerebral circulation in normal, healthy males. Since U.S.P. epinephrine has been shown to contain significant amounts of $l$-nor-epinephrine $(14,15)$, it was necessary to determine the effect of both U.S.P. epinephrine and uncontaminated synthetic $l$-epinephrine.

\section{METHOD}

The cerebral blood flow (CBF) was determined by the nitrous oxide method of Kety and Schmidt (16), as modi- 
TABLE I

The effect of 1-nor-epinephrine on cerebral circulatory functions

\begin{tabular}{|c|c|c|c|c|c|c|c|c|c|c|}
\hline \multirow[b]{2}{*}{ Subject } & \multirow[b]{2}{*}{ Age } & \multirow{2}{*}{ 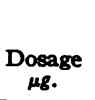 } & \multicolumn{2}{|c|}{$c c$ CBF } & \multicolumn{2}{|c|}{$\underset{m m . H g / c c . / m i n . / 100 \text { gms. }}{\text { CVR }}$} & \multicolumn{2}{|c|}{$\begin{array}{c}\text { CMR O } \\
\text { cc./min./100 gms. }\end{array}$} & \multicolumn{2}{|c|}{$\begin{array}{r}\text { MABP } \\
\mathbf{m m . H g}\end{array}$} \\
\hline & & & Control & Exper. & Control & Exper. & Control & Exper. & Control & Exper \\
\hline $\begin{array}{l}\text { H. W. } \\
\text { A. R. H. } \\
\text { H. L. B. } \\
\text { W. C. C. } \\
\text { C. W. } \\
\text { A. M. M. } \\
\text { R. B. } \\
\text { W. L. P. } \\
\text { J. F. C. } \\
\text { R. H. H. } \\
\text { J. W. B. } \\
\text { G. D. } \\
\text { R. A. P. }\end{array}$ & $\begin{array}{l}29 \\
21 \\
27 \\
40 \\
26 \\
33 \\
23 \\
32 \\
27 \\
23 \\
21 \\
36 \\
24\end{array}$ & $\begin{array}{r}1,000 \\
1,000 \\
1,400 \\
1,000 \\
800 \\
800 \\
800 \\
1,000 \\
1,000 \\
1,000 \\
700 \\
800 \\
800\end{array}$ & $\begin{array}{l}54 \\
58 \\
61 \\
67 \\
42 \\
83 \\
50 \\
45 \\
60 \\
62 \\
83 \\
68 \\
63\end{array}$ & $\begin{array}{l}49 \\
45 \\
52 \\
54 \\
33 \\
51 \\
34 \\
43 \\
43 \\
57 \\
56 \\
54 \\
59\end{array}$ & $\begin{array}{l}1.35 \\
1.36 \\
1.44 \\
1.10 \\
1.90 \\
0.81 \\
1.72 \\
1.46 \\
1.15 \\
1.16 \\
0.96 \\
1.16 \\
1.11\end{array}$ & $\begin{array}{l}2.02 \\
2.00 \\
2.09 \\
1.94 \\
3.06 \\
1.86 \\
3.09 \\
1.95 \\
2.58 \\
1.77 \\
1.64 \\
1.83 \\
1.47\end{array}$ & $\begin{array}{l}4.28 \\
4.80 \\
4.10 \\
4.11 \\
3.02 \\
4.43 \\
4.02 \\
2.98 \\
3.68 \\
3.81 \\
4.93 \\
4.07 \\
3.91\end{array}$ & $\begin{array}{l}4.35 \\
4.22 \\
5.16 \\
4.16 \\
2.86 \\
3.85 \\
2.96 \\
2.85 \\
3.07 \\
4.78 \\
3.84 \\
3.47 \\
3.85\end{array}$ & $\begin{array}{l}73 \\
79 \\
88 \\
74 \\
80 \\
67 \\
86 \\
66 \\
69 \\
72 \\
80 \\
79 \\
70\end{array}$ & $\begin{array}{r}99 \\
90 \\
109 \\
105 \\
101 \\
99 \\
105 \\
84 \\
111 \\
101 \\
92 \\
99 \\
86\end{array}$ \\
\hline $\begin{array}{l}\text { Mean } \\
\text { S.D. } \\
\text { S.E. } \\
\text { P value }\end{array}$ & 28 & & $\begin{array}{c}61 \\
11.94 \\
3.44\end{array}$ & $\begin{array}{r}48^{*} \\
8.05 \\
2.32 \\
<0.01\end{array}$ & $\begin{array}{r}1.28 \\
.28 \\
.08\end{array}$ & $\begin{array}{r}2.10^{*} \\
.48 \\
.13 \\
<0.01\end{array}$ & $\begin{array}{r}4.01 \\
.55 \\
.15\end{array}$ & $\begin{array}{r}3.80 \\
.71 \\
.20 \\
>0.20\end{array}$ & $\begin{array}{c}76 \\
6.73 \\
1.94\end{array}$ & $\begin{array}{r}99 \\
8.07 \\
2.33 \\
<0.01\end{array}$ \\
\hline
\end{tabular}

* Indicates statistically significant change.

fied by Scheinberg and Stead (17). The cerebral oxygen consumption $\left(\mathrm{CMR}_{\mathrm{O}_{2}}\right.$ ) was calculated from the $\mathrm{CBF}$ and the arterio-cerebral venous oxygen difference. The cerebral vascular resistance (CVR) was calculated from the $\mathrm{CBF}$ and the mean arterial blood pressure, which was measured directly in a peripheral artery, usually the femoral, with a damped mercury manometer. Arterial and cerebral venous blood samples were drawn simultaneously immediately before each blood flow determination. Blood oxygen and carbon dioxide content were determined manometrically (18). The cerebral blood flows were measured in the morning with the subject fasting and in the recumbent position. Immediately following a control determination, $l$-nor-epinephrine, synthetic $l$-epinephrine, ${ }^{2}$ or U.S.P. epinephrine in oil was administered intramuscularly in amounts varying from $600 \mu \mathrm{g}$. to $1,400 \mu \mathrm{g}$. Fifteen or twenty minutes later, when

2 Generous quantities of $l$-nor-epinephrine (Levophen) and $l$-epinephrine (Suprarenin) were supplied by Kenneth M. Smoot, Dept. of Medical Research, WinthropStearns, Inc.

TABLE II

The effect of 1-nor-epinephrine on arterial and cerebral venous blood constituents

\begin{tabular}{|c|c|c|c|c|c|c|c|c|c|c|c|c|c|c|}
\hline \multirow[b]{3}{*}{ Subject } & \multirow[b]{3}{*}{ Age } & \multirow{3}{*}{$\begin{array}{c}\text { Dosage } \\
\text { Mg. }\end{array}$} & \multicolumn{4}{|c|}{ Arterial blood } & \multicolumn{4}{|c|}{ Internal jugular venous blood } & \multirow{2}{*}{\multicolumn{2}{|c|}{$\begin{array}{l}\text { A-V Oz } \\
\text { Vol. \% }\end{array}$}} & \multirow{2}{*}{\multicolumn{2}{|c|}{$\begin{array}{c}\text { Oxygen extraction } \\
\text { ratio } \\
\text { ER } \mathrm{O}_{2}, \%\end{array}$}} \\
\hline & & & \multicolumn{2}{|c|}{$\begin{array}{l}\mathrm{CO}_{2} \text { content } \\
\text { vol. } \%\end{array}$} & \multicolumn{2}{|c|}{$\begin{array}{l}\mathrm{O}_{2} \text { content } \\
\text { ool. } \%\end{array}$} & \multicolumn{2}{|c|}{$\begin{array}{l}\mathrm{CO}_{2} \text { content } \\
\text { vol. } \%\end{array}$} & \multicolumn{2}{|c|}{$\begin{array}{l}\mathrm{O}_{2} \text { content } \\
\text { sol. } \%\end{array}$} & & & & \\
\hline & & & Control & Exper. & Control & Exper. & Control & Exper. & Control & Exper. & Control & Exper. & Control & Exper. \\
\hline $\begin{array}{l}\text { H. W. } \\
\text { A. R. H. } \\
\text { H. L. B. } \\
\text { W.C. C. } \\
\text { C. W. } \\
\text { A. M. M. } \\
\text { R. B. } \\
\text { W. L. P. } \\
\text { J.F. C. } \\
\text { R. H. H. } \\
\text { J. W. B. } \\
\text { G. D. } \\
\text { R. A. P. }\end{array}$ & $\begin{array}{l}29 \\
21 \\
27 \\
40 \\
26 \\
33 \\
23 \\
32 \\
27 \\
23 \\
21 \\
36 \\
24\end{array}$ & $\begin{array}{r}1,000 \\
1.000 \\
1,400 \\
1,000 \\
800 \\
800 \\
800 \\
1,000 \\
1,000 \\
1,000 \\
700 \\
800 \\
800\end{array}$ & $\begin{array}{l}42.23 \\
44.50 \\
45.48 \\
46.10 \\
44.91 \\
46.95 \\
49.26 \\
49.10 \\
47.94 \\
51.88 \\
51.41 \\
48.85 \\
47.86\end{array}$ & $\begin{array}{l}40.13 \\
42.54 \\
39.13 \\
44.39 \\
43.39 \\
43.78 \\
48.09 \\
46.22 \\
45.45 \\
47.94 \\
48.50 \\
48.82 \\
46.95\end{array}$ & $\begin{array}{l}20.07 \\
22.72 \\
20.38 \\
17.60 \\
21.25 \\
18.13 \\
20.38 \\
18.69 \\
17.97 \\
19.16 \\
18.94 \\
18.67 \\
18.36\end{array}$ & $\begin{array}{l}20.94 \\
22.75 \\
22.37 \\
19.16 \\
21.82 \\
19.14 \\
20.74 \\
19.65 \\
19.17 \\
20.26 \\
19.54 \\
19.08 \\
18.36\end{array}$ & $\begin{array}{l}\mathbf{4 9 . 6 3} \\
\mathbf{5 2 . 1 3} \\
\mathbf{5 1 . 3 1} \\
\mathbf{5 2 . 8 6} \\
\mathbf{5 1 . 6 8} \\
\mathbf{5 1 . 0 5} \\
\mathbf{5 6 . 9 6} \\
\mathbf{5 5 . 4 7} \\
\mathbf{5 3 . 3 8} \\
\mathbf{5 3 . 4 0} \\
\mathbf{5 5 . 5 4} \\
\mathbf{5 4 . 2 4} \\
\mathbf{5 3 . 0 7}\end{array}$ & $\begin{array}{l}48.81 \\
51.10 \\
48.73 \\
53.76 \\
51.37 \\
51.10 \\
56.99 \\
54.00 \\
51.69 \\
55.67 \\
55.08 \\
54.76 \\
53.45\end{array}$ & $\begin{array}{l}12.15 \\
14.44 \\
13.58 \\
11.46 \\
14.05 \\
12.79 \\
12.34 \\
12.06 \\
11.84 \\
13.00 \\
14.15 \\
12.68 \\
12.15\end{array}$ & $\begin{array}{l}12.07 \\
13.38 \\
12.43 \\
11.44 \\
13.14 \\
11.59 \\
12.01 \\
12.43 \\
12.02 \\
11.87 \\
12.69 \\
12.66 \\
11.82\end{array}$ & $\begin{array}{l}7.92 \\
8.28 \\
6.80 \\
6.14 \\
7.20 \\
5.34 \\
8.04 \\
6.63 \\
6.13 \\
6.16 \\
4.79 \\
5.99 \\
6.21\end{array}$ & $\begin{array}{l}8.87 \\
9.37 \\
9.94 \\
7.72 \\
8.68 \\
7.55 \\
8.73 \\
7.22 \\
7.15 \\
8.39 \\
6.85 \\
6.42 \\
6.54\end{array}$ & $\begin{array}{l}39 \\
36 \\
33 \\
35 \\
34 \\
29 \\
39 \\
35 \\
34 \\
32 \\
25 \\
32 \\
34\end{array}$ & $\begin{array}{l}42 \\
41 \\
44 \\
40 \\
40 \\
39 \\
42 \\
37 \\
37 \\
41 \\
35 \\
34 \\
36\end{array}$ \\
\hline $\begin{array}{l}\text { Mean } \\
\text { S.D. } \\
\text { S.E. } \\
\text { P value }\end{array}$ & 28 & & $\begin{array}{r}47.42 \\
2.66 \\
.76\end{array}$ & $\begin{array}{r}45.03^{*} \\
3.02 \\
.87 \\
<0.01\end{array}$ & $\begin{array}{r}19.41 \\
1.41 \\
.40\end{array}$ & $\begin{array}{r}20.23^{*} \\
1.33 \\
.38 \\
<0.01\end{array}$ & $\begin{array}{r}53.13 \\
1.96 \\
.56\end{array}$ & $\begin{array}{r}52.81 \\
2.46 \\
.71 \\
>0.30\end{array}$ & $\begin{array}{r}12.82 \\
.92 \\
.26\end{array}$ & $\begin{array}{c}12.27 * \\
.55 \\
.16 \\
<0.01\end{array}$ & $\begin{array}{r}6.59 \\
1.00 \\
.28\end{array}$ & $\begin{array}{r}7.96^{*} \\
1.07 \\
.31 \\
<0.01\end{array}$ & $\begin{array}{l}34 \\
3.62 \\
1.04\end{array}$ & $\begin{array}{r}39^{*} \\
2.92 \\
.84 \\
<0.01\end{array}$ \\
\hline
\end{tabular}

* Indicates statistically significant change. 
TABLE III

The effect of synthetic l-epinephrine on cerebral circulatory functions

\begin{tabular}{|c|c|c|c|c|c|c|c|c|c|c|}
\hline \multirow[b]{2}{*}{ Subject } & \multirow[b]{2}{*}{ Age } & \multirow{2}{*}{$\begin{array}{c}\text { Dosage } \\
\text { Mg. }\end{array}$} & \multicolumn{2}{|c|}{$\underset{c c . / m i n . / 100 \text { gms. }}{\text { CBF }}$} & \multicolumn{2}{|c|}{$\underset{m m . ~}{\text { Hg/cc./min. } / 100 \text { gms. }}$} & \multicolumn{2}{|c|}{$\begin{array}{c}\mathrm{CMR} \mathrm{O}_{2} \\
\text { cc./min./100 gms. }\end{array}$} & \multicolumn{2}{|c|}{$\underset{m m . H g}{\text { MABP }}$} \\
\hline & & & Control & Exper. & Control & Exper. & Control & Exper. & Control & Exper \\
\hline $\begin{array}{l}\text { B. G. T. } \\
\text { J. W. F. } \\
\text { E. B. } \\
\text { M. L. A. } \\
\text { J. E. B. } \\
\text { J. L.P. } \\
\text { B. W. M. } \\
\text { T. R. E. } \\
\text { J. W. N. } \\
\text { E. R. B. } \\
\text { C. E. B. } \\
\text { E. L. M. } \\
\text { J. M. H. } \\
\text { I. W. } \\
\text { E. L. }\end{array}$ & $\begin{array}{l}25 \\
27 \\
29 \\
33 \\
33 \\
27 \\
28 \\
33 \\
28 \\
32 \\
30 \\
35 \\
22 \\
23 \\
24\end{array}$ & $\begin{array}{r}1,000 \\
1,000 \\
600 \\
1,000 \\
800 \\
1,400 \\
1,000 \\
1,000 \\
1,000 \\
1,000 \\
1,400 \\
1,200 \\
1,000 \\
600 \\
1,200\end{array}$ & $\begin{array}{l}43 \\
57 \\
60 \\
50 \\
68 \\
49 \\
68 \\
68 \\
68 \\
60 \\
69 \\
40 \\
98 \\
95 \\
95\end{array}$ & $\begin{array}{l}45 \\
64 \\
59 \\
56 \\
67 \\
49 \\
54 \\
58 \\
76 \\
54 \\
69 \\
38 \\
97 \\
78 \\
79\end{array}$ & $\begin{array}{l}1.76 \\
1.33 \\
1.27 \\
1.62 \\
1.04 \\
1.31 \\
1.04 \\
1.07 \\
1.10 \\
1.32 \\
1.23 \\
1.70 \\
0.72 \\
0.65 \\
0.87\end{array}$ & $\begin{array}{l}1.66 \\
1.07 \\
1.24 \\
1.32 \\
0.95 \\
1.27 \\
1.22 \\
1.24 \\
0.88 \\
1.35 \\
1.13 \\
1.60 \\
0.69 \\
0.78 \\
1.01\end{array}$ & $\begin{array}{l}4.20 \\
3.82 \\
3.77 \\
3.84 \\
5.20 \\
4.01 \\
3.82 \\
4.09 \\
4.39 \\
4.57 \\
3.80 \\
2.68 \\
4.51 \\
5.28 \\
4.40\end{array}$ & $\begin{array}{l}3.61 \\
4.34 \\
4.08 \\
4.35 \\
4.95 \\
\\
2.42 \\
3.34 \\
5.02 \\
3.77 \\
4.28 \\
2.56 \\
4.37 \\
4.18 \\
5.79\end{array}$ & $\begin{array}{l}76 \\
76 \\
76 \\
81 \\
71 \\
64 \\
71 \\
73 \\
75 \\
79 \\
85 \\
68 \\
71 \\
62 \\
83\end{array}$ & $\begin{array}{l}75 \\
69 \\
73 \\
74 \\
66 \\
62 \\
66 \\
72 \\
68 \\
73 \\
78 \\
61 \\
67 \\
61 \\
80\end{array}$ \\
\hline $\begin{array}{l}\text { Mean } \\
\text { S.D. } \\
\text { S.E. } \\
\text { P value }\end{array}$ & 29 & & $\begin{array}{r}66 \\
17.57 \\
4.69\end{array}$ & $\begin{array}{r}63 \\
14.73 \\
3.93 \\
>0.10\end{array}$ & $\begin{array}{r}1.20 \\
.31 \\
.08\end{array}$ & $\begin{array}{r}1.16 \\
.26 \\
.07 \\
>0.30\end{array}$ & $\begin{array}{r}4.16 \\
.60 \\
.16\end{array}$ & $\begin{array}{r}4.08 \\
.87 \\
.24 \\
>0.50\end{array}$ & $\begin{array}{c}74 \\
6.30 \\
1.68\end{array}$ & $\begin{array}{r}70^{*} \\
5.76 \\
1.54 \\
<0.01\end{array}$ \\
\hline
\end{tabular}

* Indicates statistically significant change.

the blood pressure had become stabilized, the cerebral blood flow measurement was repeated. Previous experience with the intramuscular administration of l-nor-epinephrine had shown that a consistent pressor response occurs when the site of injection is massaged immediately after the administration of the drug. The blood pressure gradually rises for 15 to 20 minutes, becomes stabi- lized at an essentially constant level for an additional 10 to 20 minutes, and then gradually returns to normal. The second cerebral blood flow in each case was measured during the stable plateau period. The mean of four blood pressure measurements made immediately preceding and four made immediately following the blood flow determination was used as the mean arterial pressure during the

TABLE IV

The effect of synthetic l-epinephrine on arterial and cerebral venous blood constituents

\begin{tabular}{|c|c|c|c|c|c|c|c|c|c|c|c|c|c|c|}
\hline \multirow[b]{3}{*}{ Subject } & \multirow[b]{3}{*}{ Age } & \multirow{3}{*}{$\begin{array}{c}\text { Dosage } \\
\text { मf. }\end{array}$} & \multicolumn{4}{|c|}{ Arterial blood } & \multicolumn{4}{|c|}{ Internal jugular venous blood } & \multirow{2}{*}{\multicolumn{2}{|c|}{$\begin{array}{l}\text { A-V } \mathrm{O}_{2} \\
\text { Vol. } \%\end{array}$}} & \multirow{2}{*}{\multicolumn{2}{|c|}{$\begin{array}{c}\text { Oxygen extraction } \\
\text { ratio } \\
\text { ER } \mathrm{O}_{\mathbf{2}}, \%\end{array}$}} \\
\hline & & & \multicolumn{2}{|c|}{$\begin{array}{l}\mathrm{CO}_{2} \text { content } \\
\text { vol. \% }\end{array}$} & \multicolumn{2}{|c|}{$\begin{array}{l}\mathrm{O}_{2} \text { content } \\
\text { vol. \% }\end{array}$} & \multicolumn{2}{|c|}{$\begin{array}{c}\mathrm{CO}_{2} \text { content } \\
\text { vol. } \%\end{array}$} & \multicolumn{2}{|c|}{$\begin{array}{l}\mathrm{O}_{2} \text { content } \\
\text { vol. \% }\end{array}$} & & & & \\
\hline & & & Control & Exper. & Control & Exper. & Control & Exper. & Control & Exper. & Control & Exper. & Control & $\overline{\text { Exper. }}$ \\
\hline $\begin{array}{l}\text { B.G.T. } \\
\text { J.W. F. } \\
\text { E. B. } \\
\text { M.L.A. } \\
\text { J. E. B. } \\
\text { J.L.P. } \\
\text { B.W. M. } \\
\text { T.R. E. } \\
\text { J. W. N. } \\
\text { E. R. B. } \\
\text { C. E. B. } \\
\text { E. L. M. } \\
\text { J.M.H. } \\
\text { I. W. } \\
\text { E. L. }\end{array}$ & $\begin{array}{l}25 \\
27 \\
29 \\
33 \\
33 \\
27 \\
28 \\
33 \\
28 \\
32 \\
30 \\
35 \\
22 \\
23 \\
24\end{array}$ & $\begin{array}{r}1,000 \\
1,000 \\
600 \\
1,000 \\
800 \\
1,400 \\
1,000 \\
1,000 \\
1,000 \\
1,000 \\
1,400 \\
1,200 \\
1,000 \\
600 \\
1,200\end{array}$ & $\begin{array}{l}44.20 \\
43.59 \\
47.23 \\
47.73 \\
45.31 \\
47.18 \\
46.20 \\
43.34 \\
49.30 \\
46.33 \\
48.46 \\
44.37 \\
47.21 \\
48.25 \\
42.54\end{array}$ & $\begin{array}{l}45.35 \\
41.27 \\
46.77 \\
47.81 \\
46.19 \\
\\
45.52 \\
46.01 \\
50.10 \\
46.27 \\
47.50 \\
43.16 \\
48.35 \\
49.58 \\
42.28\end{array}$ & $\begin{array}{l}21.80 \\
18.35 \\
18.15 \\
20.09 \\
21.15 \\
21.27 \\
20.31 \\
17.95 \\
18.01 \\
20.98 \\
17.26 \\
15.50 \\
17.73 \\
17.72 \\
17.85\end{array}$ & $\begin{array}{l}21.72 \\
18.53 \\
18.42 \\
19.72 \\
21.46 \\
\\
20.57 \\
17.95 \\
17.88 \\
21.01 \\
17.52 \\
16.25 \\
17.69 \\
17.89 \\
18.22\end{array}$ & $\begin{array}{l}\mathbf{5 3 . 2 3} \\
49.73 \\
52.73 \\
55.05 \\
52.66 \\
55.49 \\
51.10 \\
49.48 \\
55.64 \\
53.43 \\
53.89 \\
50.79 \\
52.11 \\
53.12 \\
47.59\end{array}$ & $\begin{array}{l}\mathbf{5 3 . 2 5} \\
48.26 \\
\mathbf{5 3 . 7 6} \\
\mathbf{5 5 . 7 0} \\
\mathbf{5 3 . 5 9} \\
\\
\mathbf{5 0 . 8 7} \\
\mathbf{5 1 . 1 2} \\
\mathbf{5 6 . 4 4} \\
\mathbf{5 3 . 1 8} \\
\mathbf{5 3 . 8 0} \\
49.50 \\
\mathbf{5 3 . 4 4} \\
\mathbf{5 5 . 1 8} \\
\mathbf{5 0 . 1 7}\end{array}$ & $\begin{array}{r}12.03 \\
11.65 \\
11.87 \\
12.40 \\
13.50 \\
13.07 \\
14.68 \\
11.93 \\
11.54 \\
13.36 \\
11.75 \\
8.80 \\
12.92 \\
12.16 \\
13.21\end{array}$ & $\begin{array}{r}13.69 \\
11.75 \\
11.51 \\
11.95 \\
13.77 \\
\\
16.08 \\
12.19 \\
11.28 \\
14.03 \\
11.31 \\
9.50 \\
13.19 \\
12.52 \\
10.89\end{array}$ & $\begin{array}{l}9.77 \\
6.70 \\
6.28 \\
7.69 \\
7.65 \\
8.20 \\
5.63 \\
6.02 \\
6.47 \\
7.62 \\
5.51 \\
6.70 \\
4.81 \\
5.56 \\
4.64\end{array}$ & $\begin{array}{l}8.03 \\
6.78 \\
6.91 \\
7.77 \\
7.69 \\
\\
4.49 \\
5.76 \\
6.60 \\
6.98 \\
6.21 \\
6.75 \\
4.50 \\
5.37 \\
7.33\end{array}$ & $\begin{array}{l}45 \\
37 \\
35 \\
38 \\
36 \\
38 \\
28 \\
34 \\
36 \\
36 \\
32 \\
43 \\
27 \\
31 \\
26\end{array}$ & $\begin{array}{l}37 \\
37 \\
38 \\
39 \\
36 \\
\\
22 \\
32 \\
37 \\
33 \\
35 \\
42 \\
25 \\
30 \\
40\end{array}$ \\
\hline $\begin{array}{l}\text { Mean } \\
\text { S.D. } \\
\text { S.E. } \\
\text { P value }\end{array}$ & 29 & & $\begin{array}{r}46.08 \\
2.01 \\
.53\end{array}$ & $\begin{array}{r}46.15 \\
2.47 \\
.68 \\
>0.50\end{array}$ & $\begin{array}{r}18.94 \\
1.77 \\
.47\end{array}$ & $\begin{array}{r}18.92 \\
1.61 \\
.44 \\
>0.05\end{array}$ & $\begin{array}{r}52.40 \\
2.23 \\
.59\end{array}$ & $\begin{array}{r}52.73 \\
2.32 \\
.64 \\
>0.10\end{array}$ & $\begin{array}{r}12.32 \\
1.26 \\
.33\end{array}$ & $\begin{array}{r}12.40 \\
1.58 \\
.43 \\
>0.50\end{array}$ & $\begin{array}{r}6.62 \\
1.33 \\
.35\end{array}$ & $\begin{array}{r}6.51 \\
1.08 \\
.30 \\
>0.50\end{array}$ & $\begin{array}{c}35 \\
5.22 \\
1.39\end{array}$ & $\begin{array}{r}35 \\
5.47 \\
1.51 \\
>0.50\end{array}$ \\
\hline
\end{tabular}

\footnotetext{
* Indicates statistically significant change.
} 
TABLE V

The effect of U.S.P. epinephrine on cerebral circulatory functions

\begin{tabular}{|c|c|c|c|c|c|c|c|c|c|c|}
\hline \multirow[b]{2}{*}{ Subject } & \multirow[b]{2}{*}{ Age } & \multirow{2}{*}{$\begin{array}{c}\text { Dosage } \\
\text { Mg. }\end{array}$} & \multicolumn{2}{|c|}{$\underset{c c . / m i n . / 100 \mathrm{gms} .}{\text { CBF }}$} & \multicolumn{2}{|c|}{$\underset{m m . H_{g} / c c . / m i n . / 100 \text { gms. }}{\operatorname{CVR}}$} & \multicolumn{2}{|c|}{$\underset{c c . / m i n . / 100 \text { gms. }}{\text { CMR O }}$} & \multicolumn{2}{|c|}{$\underset{m m . H g}{\text { MABP }}$} \\
\hline & & & Control & Exper. & Control & Exper. & Control & Exper. & Control & Exper. \\
\hline $\begin{array}{l}\text { C. E. L. } \\
\text { J. B. H. } \\
\text { J.C. H. } \\
\text { B. E. C. } \\
\text { W.M. J. } \\
\text { J. J. } \\
\text { R.A.A. } \\
\text { E. F. J. } \\
\text { E. L. C. } \\
\text { N. G. W. } \\
\text { J. M. M. } \\
\text { A. A. B. } \\
\text { C. D. D. }\end{array}$ & $\begin{array}{l}36 \\
39 \\
30 \\
38 \\
34 \\
32 \\
30 \\
31 \\
28 \\
41 \\
25 \\
34 \\
25\end{array}$ & $\begin{array}{r}800 \\
1,000 \\
800 \\
800 \\
1,200 \\
1,200 \\
1,000 \\
1,000 \\
1,000 \\
1,000 \\
1,000 \\
1,000 \\
1,000\end{array}$ & $\begin{array}{l}79 \\
42 \\
58 \\
69 \\
97 \\
67 \\
80 \\
85 \\
71 \\
76 \\
69 \\
50 \\
82\end{array}$ & $\begin{array}{l}59 \\
50 \\
61 \\
51 \\
84 \\
69 \\
84 \\
94 \\
63 \\
73 \\
64 \\
49 \\
73\end{array}$ & $\begin{array}{l}0.89 \\
1.83 \\
1.28 \\
1.04 \\
0.76 \\
1.00 \\
1.01 \\
0.99 \\
0.95 \\
1.09 \\
1.28 \\
1.74 \\
0.96\end{array}$ & $\begin{array}{l}1.20 \\
1.34 \\
1.05 \\
1.43 \\
0.82 \\
0.90 \\
0.88 \\
0.84 \\
1.00 \\
0.99 \\
1.28 \\
1.69 \\
1.05\end{array}$ & $\begin{array}{l}4.78 \\
2.73 \\
2.91 \\
5.44 \\
5.07 \\
3.93 \\
3.76 \\
5.28 \\
4.54 \\
4.59 \\
4.02 \\
3.62\end{array}$ & $\begin{array}{l}4.52 \\
3.38 \\
3.58 \\
4.12 \\
4.10 \\
3.53 \\
5.39 \\
5.59 \\
3.57 \\
4.41 \\
4.18 \\
3.26 \\
4.67\end{array}$ & $\begin{array}{l}70 \\
77 \\
74 \\
72 \\
74 \\
67 \\
81 \\
84 \\
68 \\
83 \\
89 \\
87 \\
79\end{array}$ & $\begin{array}{l}71 \\
67 \\
64 \\
73 \\
69 \\
62 \\
74 \\
79 \\
64 \\
72 \\
92 \\
83 \\
77\end{array}$ \\
\hline $\begin{array}{l}\text { Mean } \\
\text { S.D. } \\
\text { S.E. } \\
\text { P value }\end{array}$ & 33 & & $\begin{array}{r}71 \\
14.24 \\
4.11\end{array}$ & $\begin{array}{r}67 \\
13.52 \\
3.90 \\
>0.10\end{array}$ & $\begin{array}{r}1.14 \\
.30 \\
.08\end{array}$ & $\begin{array}{r}1.11 \\
.25 \\
.07 \\
>0.50\end{array}$ & $\begin{array}{r}4.22 \\
.84 \\
.25\end{array}$ & $\begin{array}{r}4.18 \\
.70 \\
>.20 \\
>0.50\end{array}$ & $\begin{array}{l}77 \\
6.91 \\
1.99\end{array}$ & $\begin{array}{r}73^{*} \\
8.10 \\
2.33 \\
<0.01\end{array}$ \\
\hline
\end{tabular}

* Indicates statistically significant change.

procedure. Blood pressure values before and after measurement of CBF did not vary more than a few millimeters in any case.

\section{RESULTS}

l-Nor-epinephrine. The data (Tables I and II) indicate that $l$-nor-epinephrine is a potent cerebral vasoconstrictor. The disproportionate increase (64 per cent) in calculated mean CVR ( $p=<0.01)$ above MABP ( 31 per cent), eventuates in a 21 per cent reduction in CBF $(p=<.01)$. Nevertheless, the $\mathrm{CMR}_{\mathrm{O}_{2}}$ is unaffected because the fall in $\mathrm{CBF}$ is compensated by an increase in the oxygen extraction ratio ( 34 to 39 per cent) and in mean arterio-cerebral venous oxygen difference (6.59 vol. per cent to 7.96 vol. per cent $)(p=<.01)$. The increase in $A-V$ oxygen difference is the re-

TABLE VI

The effect of U.S.P. epinephrine on arterial and cerebral venous blood constituents

\begin{tabular}{|c|c|c|c|c|c|c|c|c|c|c|c|c|c|c|}
\hline \multirow[b]{3}{*}{ Subject } & \multirow[b]{3}{*}{ Age } & \multirow{3}{*}{$\begin{array}{c}\text { Dosage } \\
\text { Mg. }\end{array}$} & \multicolumn{4}{|c|}{ Arterial blood } & \multicolumn{4}{|c|}{ Internal jugular venous blood } & \multirow{2}{*}{\multicolumn{2}{|c|}{$\begin{array}{l}\text { A-V } \mathrm{O}_{2} \\
\text { Vol. \% }\end{array}$}} & \multirow{2}{*}{\multicolumn{2}{|c|}{$\begin{array}{c}\text { Oxygen extraction } \\
\text { ratio } \\
\text { ER } \mathrm{O}_{2}, \%\end{array}$}} \\
\hline & & & \multicolumn{2}{|c|}{$\begin{array}{l}\mathrm{CO}_{2} \text { content } \\
\text { sol. } \%\end{array}$} & \multicolumn{2}{|c|}{$\begin{array}{c}\mathrm{O}_{2} \text { content } \\
m 01 . \%\end{array}$} & \multicolumn{2}{|c|}{$\begin{array}{c}\mathrm{CO}_{2} \text { content } \\
\text { vol. } \%\end{array}$} & \multicolumn{2}{|c|}{$\begin{array}{c}\mathrm{O}_{2} \text { content } \\
\text { vol. } \%\end{array}$} & & & & \\
\hline & & & Control & Exper. & Control & Exper. & Control & Exper. & Control & Exper. & Control & Exper. & Control & Exper. \\
\hline $\begin{array}{l}\text { C. E. L. } \\
\text { J. B. H. } \\
\text { J. C. H. } \\
\text { B. E. C. } \\
\text { W. M. J. } \\
\text { J. J. } \\
\text { R. A. A. } \\
\text { E. F. J. } \\
\text { E. L. C. } \\
\text { N. G. W. } \\
\text { J. M. M. } \\
\text { A. A. B. } \\
\text { C. D. D. }\end{array}$ & $\begin{array}{l}36 \\
39 \\
30 \\
38 \\
34 \\
32 \\
30 \\
31 \\
28 \\
41 \\
25 \\
34 \\
25\end{array}$ & $\begin{array}{r}800 \\
1,000 \\
800 \\
800 \\
1,200 \\
1,200 \\
1,000 \\
1,000 \\
1,000 \\
1,000 \\
1,000 \\
1,000 \\
1,000\end{array}$ & $\begin{array}{l}47.37 \\
47.75 \\
46.74 \\
47.83 \\
48.17 \\
39.38 \\
52.10 \\
47.39 \\
44.16 \\
48.32 \\
48.46 \\
46.75\end{array}$ & $\begin{array}{l}46.24 \\
49.48 \\
47.50 \\
48.73 \\
49.68 \\
40.27 \\
50.54 \\
48.55 \\
46.91 \\
49.09 \\
49.00 \\
46.90 \\
48.03\end{array}$ & $\begin{array}{l}17.93 \\
18.80 \\
17.03 \\
20.36 \\
17.85 \\
15.65 \\
18.38 \\
20.00 \\
16.34 \\
16.75 \\
16.68 \\
20.23\end{array}$ & $\begin{array}{l}18.63 \\
18.46 \\
16.71 \\
20.38 \\
17.70 \\
15.67 \\
20.02 \\
19.95 \\
15.37 \\
16.32 \\
16.63 \\
20.13 \\
21.10\end{array}$ & $\begin{array}{l}53.77 \\
54.77 \\
51.88 \\
55.93 \\
53.62 \\
45.74 \\
56.75 \\
54.02 \\
51.20 \\
54.80 \\
54.49 \\
53.77\end{array}$ & $\begin{array}{l}\mathbf{5 2 . 7 3} \\
\mathbf{5 6 . 0 6} \\
\mathbf{5 3 . 7 2} \\
\mathbf{5 6 . 4 5} \\
\mathbf{5 5 . 3 4} \\
\mathbf{4 5 . 5 3} \\
\mathbf{5 6 . 5 5} \\
\mathbf{5 4 . 4 0} \\
\mathbf{5 2 . 6 0} \\
\mathbf{5 6 . 0 0} \\
\mathbf{5 5 . 9 3} \\
\mathbf{5 4 . 4 6} \\
\mathbf{5 3 . 8 2}\end{array}$ & $\begin{array}{r}11.88 \\
12.30 \\
12.01 \\
12.47 \\
12.62 \\
9.78 \\
13.67 \\
13.79 \\
9.94 \\
10.71 \\
10.84 \\
13.03\end{array}$ & $\begin{array}{r}11.91 \\
11.71 \\
10.84 \\
12.30 \\
12.82 \\
10.56 \\
13.60 \\
14.00 \\
9.69 \\
10.28 \\
10.10 \\
13.48 \\
14.70\end{array}$ & $\begin{array}{l}6.05 \\
6.50 \\
5.02 \\
7.89 \\
5.23 \\
5.87 \\
4.71 \\
6.21 \\
6.40 \\
6.04 \\
5.84 \\
7.23\end{array}$ & $\begin{array}{l}6.72 \\
6.75 \\
5.87 \\
8.08 \\
4.88 \\
5.11 \\
6.42 \\
5.95 \\
5.68 \\
6.04 \\
6.53 \\
6.65 \\
6.40\end{array}$ & $\begin{array}{l}34 \\
35 \\
29 \\
39 \\
29 \\
38 \\
26 \\
31 \\
39 \\
36 \\
35 \\
36\end{array}$ & $\begin{array}{l}36 \\
37 \\
35 \\
40 \\
28 \\
33 \\
32 \\
30 \\
37 \\
37 \\
39 \\
33 \\
30\end{array}$ \\
\hline $\begin{array}{l}\text { Mean } \\
\text { S.D. } \\
\text { S.E. } \\
\text { P value }\end{array}$ & 33 & & $\begin{array}{r}47.04 \\
2.87 \\
.86\end{array}$ & $\begin{array}{r}47.76 \\
2.46 \\
.71 \\
>0.05\end{array}$ & $\begin{array}{r}18.00 \\
1.52 \\
.45\end{array}$ & $\begin{array}{r}18.24 \\
1.88 \\
.54 \\
>0.50\end{array}$ & $\begin{array}{r}53.40 \\
2.72 \\
.82\end{array}$ & $\begin{array}{c}54.12^{*} \\
2.79 \\
.80 \\
<0.02\end{array}$ & $\begin{array}{r}11.92 \\
1.28 \\
.38\end{array}$ & $\begin{array}{r}12.00 \\
1.57 \\
.45 \\
>0.30\end{array}$ & $\begin{array}{r}6.08 \\
.85 \\
.25\end{array}$ & $\begin{array}{r}6.24 \\
.77 \\
.22 \\
>0.50\end{array}$ & $\begin{array}{l}34 \\
4.07 \\
1.22\end{array}$ & $\begin{array}{c}34 \\
3.58 \\
1.03 \\
>0.40\end{array}$ \\
\hline
\end{tabular}

* Indicates statistically significant change. 
sult of a significant increase in arterial $\mathrm{O}_{2}$ content (19.41 vol. per cent to 20.23 vol. per cent) and a decrease in cerebral venous oxygen content (12.82 vol. per cent to 12.27 vol. per cent). Mean arterial $\mathrm{CO}_{2}$ content decreased significantly (47.42 vol. per cent to 45.03 vol. per cent) apparently as a result of mild hyperventilation.

Synthetic l-epinephrine. In contrast to l-norepinephrine, this substance had no significant effect on mean cerebral hemodynamics or metabolism (Table III) ; nor were any changes in blood gases noted (Table IV). The only change of significance was a small but consistent reduction in MABP from $74 \mathrm{~mm}$. $\mathrm{Hg}$ to $70 \mathrm{~mm}$. $\mathrm{Hg}$ ( $\mathrm{p}=<$ 0.01).

U.S.P. epinephrine. The results of studies made with U.S.P. epinephrine are listed in Table V and Table VI. With one exception they are identical with the findings in the synthetic epinephrine series, i.e., a slight but significant reduction in MABP ( $77 \mathrm{~mm}$. $\mathrm{Hg}$ to $73 \mathrm{~mm}$. $\mathrm{Hg} ; \mathrm{P}=<.01$ ) without changes in $\mathrm{CBF}, \mathrm{CVR}, \mathrm{CMR}_{\mathrm{O}_{2}}$, arterial or venous oxygen content. With both synthetic and U.S.P. epinephrine there was an increase in mean cerebral venous $\mathrm{CO}_{2}$ content which on statistical analysis proved to be of significance only with U.S.P. epinephrine.

\section{DISCUSSION}

These experiments demonstrate that the administration of $l$-nor-epinephrine results in powerful cerebral vasoconstriction in normal healthy males. The increase in cerebral vascular resistance induced by this substance exceeds the accompanying increase in perfusion pressure; as a consequence, the blood flow to the brain is diminished. This decrease in CBF, however, does not eventuate in a reduction in cerebral oxygen consumption because of the compensatory increase in the extraction of oxygen from arterial blood by the brain, i.e., the arterio-cerebral venous oxygen difference increases.

The increase in cerebral vascular resistance is most likely due to a direct vasoconstricting effect of $l$-nor-epinephrine upon cerebral vessels. However, this vasoconstricting action could be augmented by changes in $\mathrm{O}_{2}$ tension, $\mathrm{CO}_{2}$ tension or $\mathrm{pH}$ of the arterial blood (19). King, Sokoloff, and Wechsler (20) have shown that the intra- venous infusion of $l$-nor-epinephrine is not accompanied by a change in $\mathrm{pH}$ of the blood. However, a rise in arterial oxygen content and a fall in arterial carbon dioxide content occurs when the drug is administered by either the intramuscular or intravenous route. These changes in blood gases are attributed to the mild hyperventilation that was observed in most of the subjects. However, they are not of sufficient magnitude to account for the marked increase in cerebral vascular resistance and could contribute only in a small way, if at all, to the total increase in cerebral vascular resistance (19). It seems very unlikely that reduced arterial $\mathrm{CO}_{2}$ tension is causally related to the cerebral vasoconstriction induced by $l$-norepinephrine although it is possible that slight changes in blood $\mathrm{CO}_{2}$ tension incident to hyperventilation might tend to augment the direct vasoconstricting effect of the drug. There is the further possibility that acute hypertension resulting from the administration of $l$-nor-epinephrine may be associated with an increase in intracranial pressure, and in this way further augment the resistance to blood flow in the brain.

These studies disclosed a striking difference between the cerebral circulation in essential hypertension and the acute hypertension induced by $l$-nor-epinephrine. In the former there are parallel increases in cerebral vascular resistance and blood pressure; the $\mathrm{CBF}$, therefore, remains unaltered (21). In the latter, the augmentation of CVR is out of proportion to the increase in blood pressure; the blood flow is reduced. This does not, however, constitute strong evidence against the suggestion that $l$-nor-epinephrine may be involved in the pathogenesis of essential hypertenion, since the hypertension noted in this study was acute and the state of the cerebral circulation under these circumstances not necessarily comparable to that in chronic essential hypertension. Furthermore, there is the possibility, indicated above, that indirect and secondary factors not present in essential hypertension may augment the increase in cerebral vascular resistance consequent to the acute administration of $l$-nor-epinephrine.

The demonstration that the pressor response of $l$-nor-epinephrine is accompanied by a reduction in cerebral blood flow in normotensive subjects suggests that this drug should be used with cau- 
tion as a therapeutic agent in acute hypotensive states when a reduction in blood flow to the brain may not be desirable.

Other vital segments of the circulation have been shown to be similarly affected by the administration of this substance. Renal and hepatic blood flow are diminished and calculated renal and splanchnic vascular resistance increased during the administration of nor-epinephrine (22-25). Until it can be shown that the cerebral, as well as the renal and hepatic, circulations in patients with acute hypotension respond differently to $l$-norepinephrine than in normotensive subjects, its use here should be considered potentially harmful.

These studies with epinephrine confirm the previous assumption that the quantities of norepinephrine present in U.S.P. epinephrine do not alter the effect of the latter (5), and they demonstrate yet another difference in the hemodynamic effects of nor-epinephrine and epinephrine. The intramuscular administration of epinephrine leads to a slight reduction in mean arterial blood pressure but does not alter cerebral vascular resistance, cerebral blood flow or cerebral oxygen consumption. These findings are in disagreement with those of King, Sokoloff, and Wechsler (20) who found that the intravenous infusion of epinephrine was accompanied by an increase in blood pressure, cerebral blood flow and cerebral oxygen consumption; cerebral vascular resistance was not changed. The differences in blood flow and blood pressure responses can be adequately accounted for by the difference in mode of administration of epinephrine in the two studies. Our experimental blood flows were determined 25 to 30 minutes after the intramuscular administration of epinephrine when there were clear-cut clinical manifestations of its effect such as tremulousness, tachycardia, palpitation, pallor, excitement, and apprehension; no special attempt was made to produce a pressor response in the subjects. In the studies of King, Sokoloff, and Wechsler (20) epinephrine was administered by intravenous infusion, the rate of which was adjusted to provide a pressor response before the experimental studies were done. The concentration of circulating epinephrine obtained as a result of the slow absorption from an intramuscular deposit of epinephrine in oil was no doubt inadequate to cause an increase in cardiac output of sufficient magnitude to overcome the over-all vasodilating effect of epinephrine. Since epinephrine by either route of administration does not alter cerebral vascular resistance, its effect upon the blood flow to the brain will depend directly upon its effect upon blood pressure.

The results of the two studies regarding cerebral oxygen utilization, however, cannot be attributed to the difference in mode of administration. Excitement, apprehension, palpitation, tremulousness, pallor, etc., occurred in most of the subjects after the intramuscular administration of $l$-epinephrine and U.S.P. epinephrine, and in many instances these manifestations were quite prominent. Cerebral oxygen consumption, however, was unaffected by these agents. No change in mean $\mathrm{CMR}_{\mathrm{O}_{2}}$ was observed in groups who received either $l$-epinephrine or U.S.P. epinephrine. Nor were there individual instances in either group in which there appeared to be a relationship between changes in cerebral oxygen consumption and the severity of the subjective sensations experienced by the subjects.

The results in this respect are similar to those of Scheinberg and Stead (17) who found that cerebral blood flow and cerebral oxygen consumption were unaltered by apprehension and anxiety in normal young males, and they tend to support the suggestion of Scheinberg (26) that cerebral metabolism normally functions at nearly its maximum rate. Apprehension and anxiety, whether occurring spontaneously or induced by the intramuscular administration of epinephrine, do not appear to be associated with changes in cerebral oxygen consumption.

\section{SUMMARY}

1. The effect of the intramuscular administration of $l$-nor-epinephrine, synthetic $l$-epinephrine, and U.S.P. epinephrine on cerebral circulatory and metabolic function has been determined in normal healthy males.

2. $l$-Nor-epinephrine increases mean arterial blood pressure and reduces cerebral blood flow in normotensive males by virtue of its potent constricting effect upon cerebral blood vessels. Cerebral oxygen utilization is not altered.

3. Epinephrine, when administered intramuscularly in dosage of from $600 \mu \mathrm{g}$. to $1,400 \mu \mathrm{g}$., 
slightly lowers mean arterial blood pressure; does not affect cerebral blood flow, cerebral vascular resistance or cerebral oxygen utilization.

4. The cerebrovascular effects of synthetic $l$-epinephrine and U.S.P. epinephrine are identical.

\section{ACKNOWLEDGMENTS}

This work was done with the technical assistance of Mrs. Angeline M. Hayes and Miss Mildred T. Brennan. Mr. Tyree Loving also gave valuable assistance.

\section{REFERENCES}

1. Von Euler, U. S., and Hamberg, U., L-noradrenaline in the suprarenal medulla. Nature, London, 1949, 163,642 .

2. Bülbring, E., The methylation of noradrenaline by minced suprarenal tissue. Brit. J. Pharmacol., 1949, 4, 234.

3. Bülbring, E., and Burn, J. H., Liberation of noradrenaline from adrenal medulla by splanchnic stimulation. Nature, London, 1949, 163, 363.

4. Bülbring, E., and Burn, J. H., Formation of adrenaline from noradrenaline in the perfused suprarenal gland. Brit. J. Pharmacol., 1949, 4, 245.

5. Goldenberg, M., Faber, M., Alston, E. J., and Chargaff, E. C., Evidence for the occurrence of norepinephrine in the adrenal medulla. Science, 1949, 109, 534.

6. Goldenberg, M., Pines, K. L., Baldwin, E. DeF., Greene, D. G., and Roh, C. E., The hemodynamic response of man to nor-epinephrine and epinephrine and its relation to the problem of hypertension. Am. J. Med., 1948, 5, 792.

7. Barcroft, H., and Konzett, H., On the actions of noradrenaline and isopropyl noradrenaline on the arterial blood pressure, heart rate, and muscle blood flow in man. J. Physiol., 1949, 110, 194.

8. McChesney, E. W., McAuliff, J. P., and Blumberg, H., The hyperglycemic action of some analogs of epinephrine. Proc. Soc. Exper. Biol. \& Med., 1949, 71, 220.

9. Madison, L. L., A comparison of the anterior pituitary-adrenal cortical stimulating effect of U.S.P. epinephrine, synthetic 1-epinephrine and nor-epinephrine. J. Clin. Invest., 1950, 29, 789.

10. Holton, $P$., Noradrenaline in tumors of the adrenal medulla. J. Physiol., 1949, 108, 525.

11. Pitcairn, D. M., and Youmans, W. B., The nature of pressor substances in pheochromocytomas. Circulation, 1950, 2, 505.

12. Goldenberg, M., Apgar, V., Deterling, R., and Pines, K. L., Nor-epinephrine (arterenol, 8 Sympathin N) as a pressor drug. J. A. M. A., 1949, 140, 776.
13. Miller, A. J., and Baker, L. A., L-arterenol (Levophed(8) in the treatment of shock due to acute myocardial infarction. Arch. Int. Med., 1952, 89, 591.

14. Tullar, B. F., The separation of $L$-arterenol from natural U.S.P. epinephrine. Science, 1949, 109, 536.

15. Auerbach, M. E., and Angell, E., The determination of arterenol in epinephrine. Science, 1949, 109, 537.

16. Kety, S. S., and Schmidt, C. F., The nitrous oxide method for the quantitative determination of cerebral blood flow in man: theory, procedure and normal values. J. Clin. Invest., 1948, 27, 476.

17. Scheinberg, P., and Stead, E. A., Jr., The cerebral blood flow in male subjects as measured by the nitrous oxide technique. Normal values for blood flow, oxygen utilization, glucose utilization, and peripheral resistance, with observations on the effect of tilting and anxiety. J. Clin. Invest., 1949, 28, 1163.

18. Peters, J. A., and Van Slyke, D. D., Quantitative Clinical Chemistry. Vol. II, Methods. Baltimore, Williams and Wilkins, 1931.

19. Kety, S. S., and Schmidt, C. F., The effects of altered arterial tensions of carbon dioxide and oxygen on cerebral blood flow and cerebral oxygen consumption in normal young men. J. Clin. Invest., 1948, $27,484$.

20. King, B. D., Sokoloff, L., and Wechsler, R. L., The effects of $l$-epinephrine and $l$-nor-epinephrine upon cerebral circulation and metabolism in man. J. Clin. Invest., 1952, 31, 273.

21. Kety, S. S., Hafkenschiel, J. H., Jeffers, W. A., Leopold, I. H., and Shenkin, H. A., The blood flow, vascular resistance, and oxygen consumption of the brain in essential hypertension. J. Clin. Invest., 1948, 27, 511.

22. Smythe, C. McC., Nickel, J. F., and Bradley, S. E., The effect of epinephrine (USP), $l$-epinephrine, and $l$-norepinephrine on glomerular filtration rate, renal plasma flow, and the urinary excretion of sodium, potassium, and water in normal man. J. Clin. Invest., 1952, 31, 499.

23. Moyer, J. H., and Handley, C. A., Norepinephrine and epinephrine effect on renal hemodynamics. Circulation, 1952, 5, 91.

24. Pullman, T. N., McClure, W. W., and Windhorst, D. B., The effects of 1-nor-adrenaline on renal hemodynamics. J. Lab. \& Clin. Med., 1951, 38, 940.

25. Bearn, A. G., Billing, B., and Sherlock, S., The effect of adrenalin and noradrenalin on hepatic blood flow and splanchnic carbohydrate metabolism in man. J. Physiol., 1951, 115, 430.

26. Scheinberg, P., Cerebral circulation and metabolism in hyperthyroidism. J. Clin. Invest., 1950, 29, 1010. 